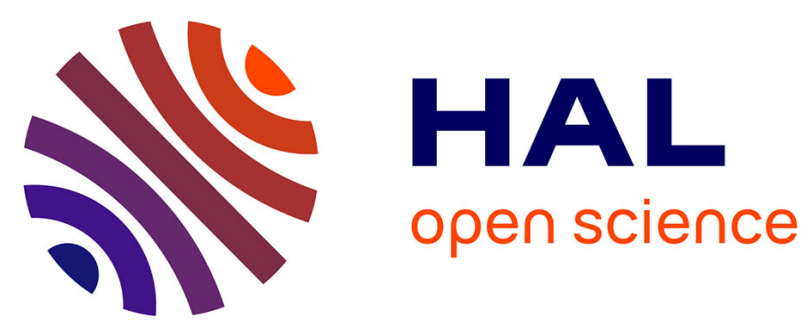

\title{
Immediate post-mastectomy breast reconstruction followed by radiotherapy: risk factors for complications
}

Didier Cowen, E. Gross, P. Rouannet, E. Teissier, S. Ellis, M. Resbeut, A. Tallet, V. Vaini Cowen, David Azria, J. M. Hannoun-Levi

\section{- To cite this version:}

Didier Cowen, E. Gross, P. Rouannet, E. Teissier, S. Ellis, et al.. Immediate post-mastectomy breast reconstruction followed by radiotherapy: risk factors for complications. Breast Cancer Research and Treatment, 2010, 121 (3), pp.627-634. 10.1007/s10549-010-0791-5 . hal-00612983

\section{HAL Id: hal-00612983 https://hal.science/hal-00612983}

Submitted on 2 Aug 2011

HAL is a multi-disciplinary open access archive for the deposit and dissemination of scientific research documents, whether they are published or not. The documents may come from teaching and research institutions in France or abroad, or from public or private research centers.
L'archive ouverte pluridisciplinaire HAL, est destinée au dépôt et à la diffusion de documents scientifiques de niveau recherche, publiés ou non, émanant des établissements d'enseignement et de recherche français ou étrangers, des laboratoires publics ou privés. 


\title{
Immediate post-mastectomy breast reconstruction followed by radiotherapy: risk factors for complications
}

\author{
Didier Cowen $\cdot$ E. Gross $\cdot$ P. Rouannet $\cdot$ \\ E. Teissier $\cdot$ S. Ellis $\cdot$ M. Resbeut $\cdot$ A. Tallet $\cdot$ \\ V. Vaini Cowen $\cdot$ D. Azria $\cdot$ J. M. Hannoun-Levi
}

Received: 21 October 2009/Accepted: 6 February 2010/Published online: 28 April 2010

(C) Springer Science+Business Media, LLC. 2010

\begin{abstract}
The objective is to prospectively determine the factors responsible for reconstruction failure and capsular contracture in mastectomized breast cancer patients who underwent immediate two-stage breast reconstruction with a tissue expander and implant, followed by radiotherapy. This is a multicenter, prospective, non-randomized study. Between February 1998 and September 2006, we prospectively examined 141 consecutive patients, each of which received an implant after mastectomy, followed by chest wall radiotherapy at $46-50$ Gy in $23-25$ fractions. Radiotherapy was delivered during immediate post-mastectomy reconstruction. Patients were evaluated by both a radiation oncologist and a surgeon 24-36 months after treatment. The median follow-up duration was 37 months. According to Baker's classification, capsular contracture was grade 0,1 , or 2 in $67.5 \%$ of cases; it was grade 3 or 4
\end{abstract}

This study was conducted on behalf of the CORS (Cercle des Oncologues Radiothérapeutes du Sud).

An invited commentary to this article can be found at doi:10.1007/s10549-010-0878-z.

D. Cowen $(\bowtie) \cdot$ E. Gross

Radiotherapy Department, Timone Hospital, rue Saint Pierre,

13005 Marseille, France

e-mail: didier.cowen@ap-hm.fr

P. Rouannet · D. Azria

Centre Val d'Aurelle, Montpellier, France

E. Teissier

Centre Azuréen de cancérologie, Mougins, France

S. Ellis

Centre Catalan d'Oncologie, Perpignan, France in $32.5 \%$ of cases. In total, 32 breast reconstruction failures required surgery. In univariate analysis, the following factors were associated with Baker grade 3 and 4 capsular contraction: adjuvant hormone therapy $(P=0.02)$, the surgeon $(P=0.04)$, and smoking $(P=0.05)$. Only one factor was significant in multivariate analysis: the surgeon $(P=0.009)$. Three factors were associated with immediate post-mastectomy breast reconstruction failure in multiple logistic regression analysis: T3 or T4 tumors $(P=0.0005)$, smoking $(P=0.001)$, and $\mathrm{pN}+$ axillary status $(P=$ $0.004)$. Patients with none, 1,2 , or all 3 factors have a probability of failure equal to $7,15.7,48.3$, and $100 \%$, respectively $\left(P=3.6 \times 10^{-6}\right)$. The model accurately predicts $80 \%$ of failures. Mastectomy, immediate reconstruction (expander followed by implant), and radiotherapy should be considered when conservative surgery is contraindicated. Three factors may be used to select patients likely to benefit from this technique with a low failure rate.

Keywords Mastectomy - Immediate reconstruction ·

Radiotherapy · Capsular contracture .

Reconstruction failure

M. Resbeut

Croix-Rouge, Toulon, France

A. Tallet

Institut Paoli-Calmettes, Marseille, France

V. V. Cowen

Polyclinique du Parc Rambot, Aix-en-Provence, France

J. M. Hannoun-Levi

Institut Antoine Lacassagne, Nice, France 


\section{Introduction}

The relevance of post-mastectomy radiotherapy (RT) of the chest wall for breast cancer has been documented in large tumors and tumors with lymph node involvement on the axillary dissection site [21-23]. RT has been shown to increase local control and overall survival in these patients [23].

The use of immediate post-mastectomy reconstruction (IPMR) after breast cancer has markedly increased in recent years. Many patients select post-mastectomy reconstruction because it reduces the psychological and cosmetic impact of the mastectomy [12]. Furthermore, the cost of immediate reconstruction is lower than that of delayed reconstruction [14].

It is now recognized that IPMR is not associated with higher carcinogenic risks than delayed reconstruction, without retarding the implementation of adjuvant treatments [1,31]. RT dose is not affected by the presence or absence of an expander, but the expander may complicate the RT technique [20].

Two reconstruction techniques are frequently performed. The first technique uses a myocutaneous flap, either from the latissimus dorsi or the rectus abdominis (transverse rectus abdominis myocutaneous [TRAM]) $[3,8]$; the second uses a temporary expander placed in the retropectoral position, which is later replaced by a permanent implant (tissue expander/implant [TE/I]). The tissue is expanded using an inflatable expander and then replaced by an implant a while after treatment. Several research teams argue that the TE/I technique when used in conjunction with postoperative irradiation leads to more frequent complications and lesser cosmetic results than the TRAM technique $[8,13]$. However, it should be noted that the TRAM technique is often contraindicated due to patient comorbidities, which creates a selection bias.

Many authors have reported that patients undergoing RT after breast reconstruction had an increased risk of complication in comparison with those undergoing reconstruction surgery without RT [3, 10, 13, 16, 18]. Yet, very few studies have established predictors of failure for either IPMR technique.

The main goal of our study was to further investigate predictors of IPMR failure with the TE/I technique followed by RT, in order to improve the selection of patients who may benefit from this technique.

\section{Materials and methods}

This was a prospective, multicenter, non-randomized study conducted between February 1998 and September 2006, including 141 patients who had undergone mastectomy for breast carcinoma followed by immediate breast reconstruction using the TE/I technique. The patients were operated on by eight surgeons from five different centers. The indications for mastectomy were tumor size, multifocality, unhealthy excision margins, or the association of an in situ and invasive carcinoma. Patients who had received conservative treatment were not included.

The standard technique consisted of modified radical mastectomy (MRM) of the entire breast tissue without areolar preservation, followed by placement of a tissue expander after detachment of the pectoralis major muscle. The MRM procedure was performed by a first surgeon, specialized in breast oncology, while the reconstruction part of the surgery was completed by a second surgeon, specialized in plastic surgery. There were no skin sparing mastectomies. After the intervention, the expander was inflated transcutaneously as quickly as possible every week, so as to rapidly reach the desired volume, when possible prior to irradiation.

During RT, the expander inflation was discontinued in order to maintain dose homogeneity throughout the treatment. Upon completion of the adjuvant treatments, the expander was replaced by a permanent implant, usually associated with areolar reconstruction or symmetrization surgery.

\section{Radiotherapy treatment}

The RT protocol uniformly delivered $46-50$ Gy in $23-25$ fractions over 5 weeks of treatment ( 2 Gy per day) using a conformational technique, after performing a dosimetric CT-scan. The dose was delivered to the ICRU point [7], with the reconstructed chest wall receiving $95-107 \%$ of the prescribed dose. No bolus was used. The irradiated areas were the homolateral chest wall, including the subclavicular and internal mammary lymph nodes to varying degrees. The definition of the areas to be irradiated was the responsibility of the radiation oncologist. The patients were treated using tangential 6-18 MV photon beams depending on dosimetry requirements.

\section{Follow-up}

The data pertaining to IPMR failure and patients' carcinogenic progression were retrieved from the medical files. Capsular contracture (CC) was assessed by both a surgeon and a radiation oncologist in a prospective fashion at 24 36 months after the initial surgery. At least one of them had been involved in the treatment of the patient. The cosmetic results were evaluated during the last consultation using a simple question: Are you satisfied with the cosmetic result of your IPMR? "Yes" and "no" were the only possible answers. On the same occasion, patients were 
given a five-page quality of life questionnaire, the results of which will be reported in a separate publication.

Complications

IPMR failure was defined as the need for a second intervention consisting in ablation or replacement of the prosthesis. CC was graded according to Baker's modified classification [25], but for a better understanding, we called Baker $1 \mathrm{a}$ and $1 \mathrm{~b}$, respectively, grade 0 and grade 1 throughout the text. Acute complications were presented partly in a previous article [27], and as they often resolved quickly, they were not presented in this report.

Univariate statistical analysis

Any reconstruction failure requiring surgery was analyzed in relation to the patient characteristics and the treatment variables. The chi-square test was used to cross examine these variables. A value of $P<0.05$ was considered statistically significant. Data were entered and processed statistically using the SEM software [17].

\section{Multivariate statistical analysis}

A multivariate analysis was conducted on the factors found significant in univariate analysis. Logistic regression was used to determine predictors of IPMR failure. The probability of IPMR failure was investigated using the predictors of failure discovered in univariate analysis.

\section{Results}

\section{Patient characteristics}

The characteristics of patients, tumors, and treatments are summarized in Table 1. The median patient age was 49.8 years (range: 25-75 years). Mean tumor size was $34 \mathrm{~mm}$, and distribution of size across population was as follows: $\mathrm{T} 1: 33 \%$; $\mathrm{T} 2<30 \mathrm{~mm}: 21.5 \%$; $\mathrm{T} 2 \geq 30 \mathrm{~mm}$ : 22\%; T3-T4: $23.5 \%$. Of the 141 patients, 103 were nonsmokers, 31 were smokers, and for seven patients this data were unavailable. Most patients $(78 \%)$ were treated with neoadjuvant or adjuvant chemotherapy (CT), and $66 \%$ with adjuvant hormone therapy (HT). Of those treated with HT, $43 \%$ were prescribed tamoxifen, $15 \%$ anti-aromatase drugs, and $7 \%$ both in sequential order. The median delay between IPMR and RT was 4.2 months in patients with CT (range 1-17 months), and 1.9 months in patients without CT (range $0.8-4.2$ months), $(P<0.0001)$. Half of the patients were postmenopausal at the beginning of their treatment $(n=71)$. The histological cancer type included
Table 1 Characteristics of patients, tumors, and treatments

\begin{tabular}{ll}
\hline Characteristics & $n(\%)$ \\
\hline Median age (years) & 49.8 \\
Menopause & $71(50.3 \%)$ \\
Smoking & $31(23.1 \%)$ \\
Multifocality & $74(52.5 \%)$ \\
Resection performed & $131(91.6 \%)$ \\
pN+ & $77(54.6 \%)$ \\
Histology & \\
Invasine ductal carcinoma & $106(74.7 \%)$ \\
Invasive lobular carcinoma & $33(23.2 \%)$ \\
Other & $2(1.4 \%)$ \\
Associated carcinoma in situ & $80(57.5 \%)$ \\
Tumor grade & \\
1 & $25(18 \%)$ \\
2 & $78(56.1 \%)$ \\
3 & $36(25.6 \%)$ \\
Chemotherapy & $110(78 \%)$ \\
Neoadjuvant & 25 \\
Adjuvant & 85 \\
ER or PR $>10 \%$ & $100(71.4 \%)$ \\
Hormone therapy & $92(66 \%)$ \\
Tamoxifen & 61 \\
Anti-aromatase & 21 \\
Tamoxifen then anti-aromatase & 10 \\
\hline &
\end{tabular}

invasive ductal carcinoma in $74.7 \%$ of cases, invasive lobular carcinoma in $23.2 \%$ of cases, and a different histological type in $1.4 \%$ of cases. For $57.5 \%$ of cases, the cancer was in situ. The tumor was multifocal at diagnosis in $52.5 \%$ of cases. Regarding tumor grade, $18 \%$ were grade $1,56 \%$ grade 2 , and $26 \%$ grade 3 . An axillary dissection was performed in $91.6 \%$ of patients, $54.6 \%$ of which presented with $\mathrm{pN}+$.

In all cases where CT was indicated, RT was delivered after CT. The delay for permanent reconstruction depended on the type of adjuvant treatment (RT alone vs. RT $+\mathrm{CT}$ ) $(P<0.0001)$.

\section{Capsular contracture}

Capsular contracture, classified according to Baker classification, was grade 0,1 , or 2 in $67.5 \%$ of cases, and grade 3 or 4 in $32.5 \%$ of cases (Table 2). Only five (3.5\%) patients presented a severe grade $4 \mathrm{CC}$. In a univariate analysis, the factors associated with Baker 3 and 4 CC were: surgeon $(P=0.04)$, adjuvant HT $(P=0.02)$, and smoking $(P=0.05)$ (Table 3$)$. In multivariate analysis, the association was significant for the surgeon $(P=0.009)$ only. 
Table 2 Late complications after IPMR using the TE/I technique followed by radiotherapy

\begin{tabular}{lc}
\hline Complication types & $n(\%)$ \\
\hline Failures & $32(22.7 \%)$ \\
Luxation & $12(8.5 \%)$ \\
Extrusion & $7(5.0 \%)$ \\
Infection & $7(5.0 \%)$ \\
Severe capsular contracture & $6(4.3 \%)$ \\
Capsular contracture according to Baker's classification \\
Grade 0 & $35(24.9 \%)$ \\
Grade 1 & $20(14.2 \%)$ \\
Grade 2 & $40(28.4 \%)$ \\
Grade 3 & $41(29.0 \%)$ \\
Grade 4 & $5(3.5 \%)$ \\
\hline
\end{tabular}

Table 3 Capsular contracture classified as Baker grade 3 and 4 (univariate analysis)

\begin{tabular}{ll}
\hline & $P$ \\
\hline Surgeon & 0.04 \\
Hormone therapy+ & 0.02 \\
Smoking & 0.05 \\
Menopause & 0.13 \\
Chemotherapy & 0.73 \\
Multifocality & 0.26 \\
Associated carcinoma in situ & 0.28 \\
Mean size S & 0.90 \\
pN+ & 0.47 \\
Mean delay between IPMR and start of radiotherapy & 0.83 \\
\hline
\end{tabular}

Reconstruction failure

IPMR failure defined as requiring exeresis or replacement surgery occurred in 32 patients (22.7\% of cases). The events that led to failure were extrusion of the prosthesis in seven (5\%), luxation in 12 (8.6\%), infection in seven (5\%), and severe CC in seven (4.3\%) patients (Table 2).

In a univariate analysis, reconstruction failure was significantly linked to smoking $(P=0.0009)$, initial tumor size $(P=0.000016)$, and positive lymph node status $(P=0.037)$ (Table 4). Patients with an A or B breast cupsize had more failures than patients with a cup size of C, D, or E $(35.9 \%$ vs. $16.7 \%, P=0.009)$. However, cup-size was missing for 32 patients and therefore was not included in the multivariate analysis because of the risk of loosing other information. The mean delay between IPMR and the start of RT was not connected with failure $(P=0.23)$. Timing of placement of the permanent implant was not associated with failure. Grade 3 or $4 \mathrm{CC}$ rates were not significantly correlated with higher failure rates $(P=0.70)$, neither were adjuvant nor neoadjuvant $\mathrm{CT}$
Table 4 IPMR failures (univariate analysis)

\begin{tabular}{ll}
\hline & $P$ \\
\hline Surgeon & 0.08 \\
Hormone therapy & 0.05 \\
Cup size & 0.009 \\
Smoking & 0.00091 \\
Menopause & 0.41 \\
Chemotherapy & 0.30 \\
Multifocal tumor & 0.93 \\
Associated carcinoma in situ & 0.33 \\
Mean tumor size & 0.000016 \\
pN+ & 0.037 \\
Baker grade 3 and 4 & 0.70 \\
Mean delay between IPMR and start of radiotherapy & 0.23 \\
\hline
\end{tabular}

$(P=0.30)$. The three positive factors established in the univariate analysis were included in the multivariate model. They were all linked to IPMR failure with relative risks (RR) of $6.1(2.2-17)$ for $\mathrm{T} 3(P=0.0005), \mathrm{RR}=5.9$ (2-17) for smoker status $(P=0.001)$, and $\mathrm{RR}=5(1.7-$ $15.5)$ for lymph node invasion $(P=0.004)$. The association of these three co-variables allowed for IPMR failure prediction, with varying relevance (Table 5): patients with none, 1,2 , or all 3 factors had a probability of failure equal to $7,15.7,48.3$, and $100 \%$, respectively $\left(P=3.6 \times 10^{-6}\right)$.

Patient status

After a median follow-up period of 37 months (range 10 107 months), 97 (68.9\%) patients were alive and in complete remission, $21(14.9 \%)$ had cancer recurrence in the form of metastases, 13 (9.2\%) were lost to follow-up, 6 $(4.3 \%)$ had died, and $4(2.8 \%)$ had local recurrences.

\section{Patient satisfaction}

When questioned about the post-IPMR cosmetic result, 51 patients stated that they were satisfied with the results of IPMR, 72 were not satisfied, and 18 provided no answer. Among the patients who responded, the satisfaction rate

Table 5 Probability of IPMR failure according to the number of predictors (smoking, T3 or larger tumors, and $\mathrm{pN}+)(P=0.0000036)$

\begin{tabular}{lllllr}
\hline Failure yes/no & \multicolumn{5}{l}{ Risk factors $(n)$} \\
\cline { 2 - 5 } & 0 & 1 & 2 & 3 & \\
\hline No & 40 & 54 & 15 & 0 & 109 \\
Yes & 3 & 10 & 14 & 5 & 32 \\
Total & 43 & 64 & 29 & 5 & 141 \\
Failure probability & $7 \%$ & $15.7 \%$ & $48.3 \%$ & $100 \%$ & \\
\hline
\end{tabular}


Table 6 Patient dissatisfaction (univariate analysis)

\begin{tabular}{ll}
\hline & $P$ \\
\hline Type of surgeon & 0.000073 \\
Hormone therapy & 0.08 \\
Smoking & 0.54 \\
Menopause & 0.0006 \\
Chemotherapy & 0.38 \\
Mean tumor size & 0.07 \\
Baker grade 3 and 4 & 0.05 \\
Failure & 0.40 \\
Delay between IPMR and initiation of radiotherapy & 0.57 \\
\hline
\end{tabular}

was $41.4 \%$. Patient insatisfaction was not the result of IPMR failure $(P=0.40)$, but was significantly related to the existence of grade 3 or grade $4 \mathrm{CC}(P=0.05)$. Patients who were not postmenopausal $(P=0.0006)$ were statistically less satisfied with IPMR. The satisfaction of each patient depended on the surgeon $(P=0.000073)$ (Table 6). The complete results of the QOL questionnaire will be reported separately.

\section{Discussion}

IPMR using the TE/I technique after breast cancer offers cosmetic and psychological advantages [12] for both the patient and the surgeon, with only a few contraindications.

Our study involved a sizeable and homogenous population of patients who underwent IPMR using the TE/I technique followed by RT. IPMR failure rate was $22.7 \%$, with a median follow-up duration of 37 months. Higher failure rates were reported by other authors (Table 7). Chawla et al. [8] found a failure rate of $48 \%$ in 18 patients who underwent IPMR with the TE/I technique. These high rates may be due to a selection bias between the two reconstruction techniques, which were compared without any randomization.
In addition, the sample size was small. Furthermore, RT was at times performed prior to the mastectomy, resulting in an inhomogeneous patient population.

Performing the reconstruction after RT raises the risk of skin fibrosis, which renders tissue expansion more difficult, even in selected patients [15]. Krueger et al. [16] from University of Michigan reported a failure rate of $37 \%$ in 19 patients. In contrast, in larger patient populations, the reported failure rates when using the TE/I technique are in accordance with data obtained in our study [2, 13, 27].

Anderson et al. reported a $4 \%$ failure rate in their series involving 50 patients. Yet these results must be interpreted with great caution, as the follow-up period was much shorter compared to the other series (28 months). Additionally, Whitfield et al. noted a low failure rate $(8 \%)$, although, in their study, RT fractionation was different (40 Gy in 15 fractions), comparable to that used in the British START trial [4]. In their series, Cordeiro et al. [10] reported an $11 \%$ failure rate in 81 patients. However, in this study, the treatment sequence differed, with irradiation delivered after the permanent implant was placed (4 weeks), while the implant was placed 4 weeks after the end of CT. Thus, the delay between surgery and RT was longer (at least 8 weeks after the end of CT). Although this delay may account to some extent for the low reconstruction failure rate, it could also jeopardize the carcinogenic efficacy of irradiation [19].

It should be noted that the failure rate we observed in our study is high, higher than that reported with the TRAM technique [8]. This high failure rate may be due to a patient selection bias, as patients with less comorbidities and sufficient intra-abdominal fat are often treated with the TRAM technique. The identification of risk factors of TE/I failure, however, may improve patient selection.

Capsular contraction after IPMR is a complication dreaded by surgeons as it has cosmetic consequences and may require re-intervention. In our series, the rate of grade 3 and 4 CCs was $32.5 \%$ (of which $3.5 \%$ were severe grade $4 \mathrm{CCs}$, which is in line with published literature). The fact
Table 7 IPMR failure with TE/ I technique followed by external radiotherapy

\begin{tabular}{lcll}
\hline Institution/first author & $n$ & $\%$ failure & Median follow-up (months) \\
\hline Fox Chase/Anderson [2] & 50 & $4 \%$ & 28 \\
IPC/Tallet [27] & 55 & $24 \%$ & 25 \\
Massachusetts/Chawla [8] & 18 & $48 \%$ & 32 \\
Columbia/Ascherman [3] & 27 & $18.5 \%$ & Not reported \\
University of Michigan/Krueger [16] & 19 & $37 \%$ & 31 \\
Long Island/Jhaveri [13] & 69 & $20 \%$ & 38 \\
Memorial/Cordeiro [10] & 81 & $11 \%$ & 34 \\
Bordet/Vanderweyer [28] & 6 & $100 \%$ complications & 64.5 \\
& & Baker grade 3 and 4 & \\
Cambridge, UK/Whitfield [30] & 41 & $8 \%$ & 51 \\
Present study & 141 & $22.7 \%$ & 36 \\
\hline
\end{tabular}


that capsular contracture seemed associated with the surgeon needs to be explained. This finding was linked mainly to one of the surgeons involved in the mastectomies, but not with the plastic surgeon who usually did the reconstructions with him. This plastic surgeon also worked with other surgeons. The explanation given by the plastic surgeon was that this particular surgeon performed a more hemorrhagic surgery and did more injury to the underlying aponevrosis. This could have an influence on vascularization and needs to be confirmed in further studies.

Evidently, the rate of CC was related to patient satisfaction with the cosmetic results $(P=0.05)$, and not with the risk of failure $(P=0.7)$. Grade 3 and $4 \mathrm{CC}$ rates were significantly related to the surgeon. Smoking tended to be associated with grade 3 and 4 CCs $(P=0.05)$, possibly due to the impact of tobacco on healing. The association with HT appears to be statistically significant, without any clear explanations. Nevertheless, CC remains difficult to assess, as illustrated by the high variance of data found in literature (17-68\%) [12, 18, 24-26]. This may be explained by differences in patient follow-up: rate of CCs increases with increasing duration of follow-up [30]. Of note is that in our series patients were assessed by both a surgeon and a radiation oncologist, of which at least one was involved in the treatment of the patient, which may have resolved major differences in patient assessments.

Besides the IPMR technique used (TRAM or TE/I), which was found to be a predictor in most studies comparing both techniques $[8,16]$, no other predictor of IPMR failure when using TE/I technique was consistently reported by most authors (Table 8). Krueger et al. [16] identified tamoxifen as a significant risk factor; for Anderson et al. [2], the type of cutaneous bolus used was a risk factor; lastly, Tallet et al. [27] and Ascherman et al. [3] found CT to be a risk factor. In our study, no cutaneous bolus was used, and CT was not found to be a significant prognosticator $(P=0.3)$.

We found three factors that were statistically linked to IPMR failure using the TE/I technique: smoking, T3-T4 tumors, and lymph node invasion. The effects of smoking on microvascularization may explain these results, as a similar relationship had already been observed for the TRAM reconstruction technique [6]. Initial tumor size and lymph node status were significant predictors, probably because of the extent of surgery in larger tumors. Unfortunately we could not go further in the analysis of the extent of lymph node dissection because the information was not reported prospectively in our database. One of the explanations of a high rate of failure could be the number of lymph nodes removed, and as to verify this, we will analyze the pathology reports in a retrospective approach and report the results later on. Histological data was not considered to be a risk factor of failure in the published literature, which requires further investigation. Breast cupsize could be an interesting result of this study, although unfortunately the information was missing in 32 patients. If one hypothesizes that larger expansion would be associated with more complications, large tumors in small breasts may require a larger skin sacrifice and therefore may be more difficult to expand. This should be investigated in further studies as well as the amount of expansion.

HT was associated with a higher failure rate, achieving borderline significance $(P=0.05)$. Other authors have observed this trend when considering complication rates [16] or cosmetic results [29]. The induction of transforming growth factor-beta (TGF- $\beta$ ), a peptide which modulates fibrosis by controlling cell proliferation, may be a possible explanation in patients receiving tamoxifen. Tamoxifen and RT were shown to be associated with TGF- $\beta$ secretion $[5,9]$. In our study, among the 92 patients who received

Table 8 Significant predictive factors of IPMR failure/complications with the TE/I technique followed by external radiotherapy (univariate analysis)

\begin{tabular}{llll}
\hline Institution/first author & Variable & Failure/complication & $P$ \\
\hline Fox Chase/Anderson [2] & Bolus type & Major complications & 0.05 \\
IPC/Tallet [27] & Chemotherapy & Failure & 0.04 \\
& Smoking & Failure & 0.01 \\
Massachusetts/Chawla [8] & IPMR type & Failure & $<0.01$ \\
Columbia/Ascherman [3] & Chemotherapy & All complications & $<0.01$ \\
University of Michigan/Krueger [16] & Tamoxifen & Failure & 0.03 \\
Long Island/Jhaveri [13] & IPMR type & Failure & 0.007 \\
Memorial/Cordeiro [10] & Not tested & Not tested & Not tested \\
Bordet/Vanderweyer [28] & Not tested & Not tested & Not tested \\
Present study & Smoking & Failure & 0.0009 \\
& Tumor size & Failure & 0.0000016 \\
& pN+ & Failure & 0.037 \\
\hline
\end{tabular}


HT, 61 were prescribed tamoxifen, whereas only 21 were treated with anti-aromatase drugs (10 in sequential treatment). The current routine use of anti-aromatase drugs as adjuvants in postmenopausal women may decrease the relevance of this observation [11].

IPMR failures were linked to local recurrences in only four cases. The procedure appears to be more complex in the case of a larger tumor mass. In fact, in large tumors, obtaining healthy margins requires the resection of a large portion of skin. Consequently, skin expansion becomes more complex, possibly leading to complications and failures.

Our study results allowed us to create a mathematical model aimed at predicting the probability of IPMR failure. According to our model, in the absence of all three identified predictors, the probability of IPMR failure is $7 \%$; with one factor, it is $15.7 \%$; with two factors, it is $48.3 \%$; with three factors, it is $100 \%$. No other authors reported similar results, likely due to insufficient sample sizes.

This model may be useful in common clinical practice prior to proposing IPMR to patients. However, there is the problem of preoperative assessment of tumor size and lymph node status, because their determination prior to the operation is difficult. When IPMR associated with RT is considered, it may be useful to accurately determine tumor size before the procedure. Preoperative assessment of lymph nodes with ultrasound-guided biopsies needs further investigation as well as the extent of lymph node dissection.

Many authors have questioned the treatment sequence and the role of RT. Some have suggested that RT prior to IPMR would improve the complication rates [26], while others stated the opposite [10]. In our study, all of the patients underwent RT after the expander was placed. RT has proved to be effective on carcinologic results when delivered after surgery.

Patient satisfaction is a difficult criterion to assess because it is subjective, and the response to a simple question may result in a low satisfaction rate, which has previously been described [8, 10, 27]. In addition, our series did not include a control group, for example, women who underwent mastectomy for intraductal carcinoma without requiring $\mathrm{RT}$. Understandably, lower satisfaction was found in patients with grade 3 or $4 \mathrm{CC}$. The patients' perception of the cosmetic result of their IPMR is a subjective factor which fluctuates with time, and the longer the follow-up period, the more reliable the outcome becomes. The IPMR insatisfaction rate in young non-postmenopausal patients may be due to the importance they attach to their appearance, in comparison to postmenopausal patients. The control of technical expertise remains a major factor, both in regard to $\mathrm{CC}$ rates and patient satisfaction. Patient dissatisfaction was part of a much larger quality of life study which is still under analysis. We cannot give a proper analysis of this dissatisfaction before all the data are investigated. The complete data will be reported separately.

\section{Conclusion}

Our study identified three predictors of IPMR failure using the TE/I technique followed by RT: smoking, T3 or T4 tumors, and axillary lymph node invasion. Two of these factors are related to histological data. Based on these three factors, we created a predictive probability model of IPMR failure with the TE/I technique. In common practice, this model may be useful when proposing the aforementioned treatment to patients scheduled to undergo mastectomy. It could potentially help the patient and surgeon with regards to the usefulness of performing IPMR with the TE/I technique. A preoperative imaging assessment of tumor size is usually required, and some information on the axillary status of the patient could help in the decision of associating IPMR with radiotherapy.

Conflict of interest None.

\section{References}

1. Allweis TM, Boisvert ME, Otero SE, Perry DJ, Dubin NH, Priebat DA (2002) Immediate reconstruction after mastectomy for breast cancer does not prolong the time to starting adjuvant chemotherapy. Am J Surg 183:218-221

2. Anderson PR, Freedman G, Nicolaou N, Sharma N, Li T, Topham $\mathrm{N}$ et al (2009) Postmastectomy chest wall radiation to a temporary tissue expander or permanent breast implant-is there a difference in complication rates? Int J Radiat Oncol Biol Phys 74:81-85

3. Ascherman JA, Hanasono MM, Newman MI, Hughes DB (2006) Implant reconstruction in breast cancer patients treated with radiation therapy. Plast Reconstr Surg 117:359-365

4. Bentzen SM, Agrawal RK, Aird EG, Barrett JM, Barrett-Lee PJ, Bentzen SM et al (2008) The UK Standardisation of Breast Radiotherapy (START) Trial B of radiotherapy hypofractionation for treatment of early breast cancer: a randomised trial. Lancet 371:1098-1107

5. Canney PA, Dean S (1990) Transforming growth factor beta: a promotor of late connective tissue injury following radiotherapy? Br J Radiol 63:620-623

6. Chang DW, Reece GP, Wang B, Robb GL, Miller MJ, Evans GR et al (2000) Effect of smoking on complications in patients undergoing free TRAM flap breast reconstruction. Plast Reconstr Surg 105:2374-2380

7. Chavaudra J, Bridier A (2001) Definition of volumes in external radiotherapy: ICRU reports 50 and 62. Cancer Radiother 5:472478

8. Chawla AK, Kachnic LA, Taghian AG, Niemierko A, Zapton DT, Powell SN (2002) Radiotherapy and breast reconstruction: complications and cosmesis with TRAM versus tissue expander/ implant. Int J Radiat Oncol Biol Phys 54:520-526

9. Colletta AA, Wakefield LM, Howell FV, van Roozendaal KE, Danielpour D, Ebbs SR et al (1990) Anti-oestrogens induce the 
secretion of active transforming growth factor beta from human fetal fibroblasts. Br J Cancer 62:405-409

10. Cordeiro PG, Pusic AL, Disa JJ, McCormick B, VanZee K (2004) Irradiation after immediate tissue expander/implant breast reconstruction: outcomes, complications, aesthetic results, and satisfaction among 156 patients. Plast Reconstr Surg 113:877-881

11. Forbes JF, Cuzick J, Buzdar A, Howell A, Tobias JS, Baum M (2008) Effect of anastrozole and tamoxifen as adjuvant treatment for early-stage breast cancer: 100-month analysis of the ATAC trial. Lancet Oncol 9:45-53

12. Harcourt DM, Rumsey NJ, Ambler NR, Cawthorn SJ, Reid CD, Maddox PR et al (2003) The psychological effect of mastectomy with or without breast reconstruction: a prospective, multicenter study. Plast Reconstr Surg 111:1060-1068

13. Jhaveri JD, Rush SC, Kostroff K, Derisi D, Farber LA, Maurer VE et al (2008) Clinical outcomes of postmastectomy radiation therapy after immediate breast reconstruction. Int J Radiat Oncol Biol Phys 72:859-865

14. Khoo A, Kroll SS, Reece GP, Miller MJ, Evans GR, Robb GL et al (1998) A comparison of resource costs of immediate and delayed breast reconstruction. Plast Reconstr Surg 101:964-968 discussion 969-970

15. Kraemer O, Andersen M, Siim E (1996) Breast reconstruction and tissue expansion in irradiated versus not irradiated women after mastectomy. Scand J Plast Reconstr Surg Hand Surg 30:201-206

16. Krueger EA, Wilkins EG, Strawderman M, Cederna P, Goldfarb S, Vicini FA et al (2001) Complications and patient satisfaction following expander/implant breast reconstruction with and without radiotherapy. Int J Radiat Oncol Biol Phys 49:713-721

17. Kwiatkowski F, Girard M, Hacene K, Berlie J (2000) Sem: a suitable statistical software adapted for research in oncology. Bull Cancer 87:715-721

18. Mehta VK, Goffinet D (2004) Postmastectomy radiation therapy after TRAM flap breast reconstruction. Breast J 10:118-122

19. Mikeljevic JS, Haward R, Johnston C, Crellin A, Dodwell D, Jones A et al (2004) Trends in postoperative radiotherapy delay and the effect on survival in breast cancer patients treated with conservation surgery. Br J Cancer 90:1343-1348

20. Motwani SB, Strom EA, Schechter NR, Butler CE, Lee GK, Langstein $\mathrm{HN}$ et al (2006) The impact of immediate breast reconstruction on the technical delivery of postmastectomy radiotherapy. Int J Radiat Oncol Biol Phys 66:76-82

21. Overgaard M, Hansen PS, Overgaard J, Rose C, Andersson M, Bach $F$ et al (1997) Postoperative radiotherapy in high-risk premenopausal women with breast cancer who receive adjuvant chemotherapy. Danish Breast Cancer Cooperative Group 82b Trial. N Engl J Med 337:949-955

22. Overgaard M, Jensen MB, Overgaard J, Hansen PS, Rose C, Andersson $\mathrm{M}$ et al (1999) Postoperative radiotherapy in high-risk postmenopausal breast-cancer patients given adjuvant tamoxifen: Danish Breast Cancer Cooperative Group DBCG 82c randomised trial. Lancet 353:1641-1648

23. Ragaz J, Jackson SM, Le N, Plenderleith IH, Spinelli JJ, Basco VE et al (1997) Adjuvant radiotherapy and chemotherapy in node-positive premenopausal women with breast cancer. N Engl J Med 337:956-962

24. Ringberg A, Tengrup I, Aspegren K, Palmer B (1999) Immediate breast reconstruction after mastectomy for cancer. Eur J Surg Oncol 25:470-476

25. Spear SL, Baker JL Jr (1995) Classification of capsular contracture after prosthetic breast reconstruction. Plast Reconstr Surg 96:1119-1123 discussion 1124

26. Spear SL, Majidian A (1998) Immediate breast reconstruction in two stages using textured, integrated-valve tissue expanders and breast implants: a retrospective review of 171 consecutive breast reconstructions from 1989 to 1996. Plast Reconstr Surg 101:53-63

27. Tallet AV, Salem N, Moutardier V, Ananian P, Braud AC, Zalta $\mathrm{R}$ et al (2003) Radiotherapy and immediate two-stage breast reconstruction with a tissue expander and implant: complications and esthetic results. Int J Radiat Oncol Biol Phys 57:136-142

28. Vandeweyer E, Deraemaecker R (2000) Radiation therapy after immediate breast reconstruction with implants. Plast Reconstr Surg 106:56-58 discussion 59-60

29. Wazer DE, Morr J, Erban JK, Schmid CH, Ruthazer R, SchmidtUllrich RK (1997) The effects of postradiation treatment with tamoxifen on local control and cosmetic outcome in the conservatively treated breast. Cancer 80:732-740

30. Whitfield GA, Horan G, Irwin MS, Malata CM, Wishart GC, Wilson CB (2009) Incidence of severe capsular contracture following implant-based immediate breast reconstruction with or without postoperative chest wall radiotherapy using 40 Gray in 15 fractions. Radiother Oncol 90:141-147

31. Wright JL, Cordeiro PG, Ben-Porat L, Van Zee KJ, Hudis C, Beal K (2008) Mastectomy with immediate expander-implant reconstruction, adjuvant chemotherapy, and radiation for stage II-III breast cancer: treatment intervals and clinical outcomes. Int J Radiat Oncol Biol Phys 70:43-50 